\title{
QoS routing in ad-hoc networks using GA and multi-objective optimization
}

\author{
Admir Barolli ${ }^{\mathrm{a}, *}$, Evjola Spaho ${ }^{\mathrm{b}}$, Leonard Barolli ${ }^{\mathrm{c}}$, Fatos Xhafa $^{\mathrm{d}}$ and Makoto Takizawa ${ }^{\mathrm{a}}$ \\ ${ }^{a}$ Department of Computers and Information Science, Seikei University, Tokyo, Japan \\ ${ }^{\mathrm{b}}$ Graduate School of Engineering, Fukuoka Institute of Technology (FIT), Fukuoka, Japan \\ ${ }^{\mathrm{c}}$ Department of Information and Communication Engineering, Fukuoka Institute of Technology (FIT), \\ Fukuoka, Japan \\ ${ }^{\mathrm{d}}$ Department of Languages and Informatics Systems, Technical University of Catalonia, Jordi Girona \\ 1-3, Barcelona, Spain
}

\begin{abstract}
Much work has been done on routing in Ad-hoc networks, but the proposed routing solutions only deal with the best effort data traffic. Connections with Quality of Service (QoS) requirements, such as voice channels with delay and bandwidth constraints, are not supported. The QoS routing has been receiving increasingly intensive attention, but searching for the shortest path with many metrics is an NP-complete problem. For this reason, approximated solutions and heuristic algorithms should be developed for multi-path constraints QoS routing. Also, the routing methods should be adaptive, flexible, and intelligent. In this paper, we use Genetic Algorithms (GAs) and multi-objective optimization for QoS routing in Ad-hoc Networks. In order to reduce the search space of GA, we implemented a search space reduction algorithm, which reduces the search space for GAMAN (GA-based routing algorithm for Mobile Ad-hoc Networks) to find a new route. We evaluate the performance of GAMAN by computer simulations and show that GAMAN has better behaviour than GLBR (Genetic Load Balancing Routing).
\end{abstract}

\section{Introduction}

The wireless mobile networks and devices are becoming increasingly popular and they provide users access to information and communication any-time and anywhere. The conventional wireless networks are often connected to a wired network (e.g. ATM or Internet). This kind of wireless network requires a fixed wire-line backbone infrastructure. All mobile hosts in a communication cell can reach a base station on the wire-line networks in one-hop radio transmission. In contrast, the class of Ad-hoc networks do not use any fixed infrastructure. The nodes of Ad-hoc networks intercommunicate through single-hop and multi-hop paths in a peer-to-peer fashion. Intermediate nodes between two pairs of communication nodes act as routers. Thus the nodes operate both as hosts and routers. The nodes are mobile, so the creation of routing paths is affected by the addition and deletion of nodes. The topology of the network may change rapidly and unexpectedly.

Much work has been done on routing in Ad-hoc networks [1,2]. Many protocols and algorithms such as Destination-Sequenced Distance-Vector (DSDV) protocol, cluster-based routing algorithms, Dynamic Source Routing (DSR) protocol, Ad-hoc On-demand Distance-Vector (AODV) protocol, Zone Routing Protocol (ZRP), Temporally Ordered Routing Algorithm (TORA), and Associative Bit Routing (ABR)

\footnotetext{
${ }^{*}$ Corresponding author. E-mail: admir.barolli@gmail.com.
} 
have been proposed. The emphasis has been on providing the shortest path and achieving a high degree of availability in a dynamic environment where the network topology changes fast. However, all the above routing solutions only deal with the best effort data traffic.

The QoS routing has been receiving increasingly intensive attention in the wire-line network domain [3, 4]. The routing strategies can be classified into three classes: source, distributed and hierarchical routing. However, these QoS routing algorithms can not be applied directly to Ad-hoc networks, because of the bandwidth constraints and dynamic network topology of Ad-hoc networks.

Recently, because of the rising popularity of multimedia applications and potential commercial usages of Ad-hoc networks, QoS support in Ad-hoc networks has become an unavoidable task. To support QoS, the link state information such as delay, bandwidth, cost, loss rate, and error rate in the network should be available and manageable. But, getting and managing the link state information in Ad-hoc networks is very difficult because the quality of wireless link may change with the surrounding circumstances. Furthermore, the resource limitation and the mobility of hosts make things more complicated. The challenge we face is to implement complex QoS functionality with limited available resources in a dynamic environment.

In the literature, the research on QoS support in Ad-hoc networks includes QoS models [5,6], QoS resource reservation signalling [7], QoS Medium Access Control (MAC) [8], and QoS routing [9-11]. In this paper, we will survey only QoS routing problem in Ad-hoc networks. The QoS routing searches for a path with enough resources for QoS requirements. The QoS metrics could be additive, concave or productive. It is proved that if QoS contains at least two additive metrics, then the QoS routing is a NP complete problem [11]. Therefore, searching for the shortest path with minimal cost and finding delay constrained least-cost paths are NP-complete problems. For this reason, approximated solutions and heuristic algorithms should be developed for multi-path constraints QoS routing. Also, to cope with changing of Ad-hoc networks topology, routing methods should be adaptive, flexible, and intelligent.

Use of intelligent algorithms based on Fuzzy Logic (FL), Neural Networks (NNs) and Genetic Algorithms (GAs) can prove to be efficient for traffic control in telecommunication networks [12,?,?,?]. In difference from non-linear programming methods, GA, FL and NNs are heuristic methods which use explicit rules to find feasible routes. The GA uses the genetic operators for optimization. By finding good values of crossover probability, mutation probability, and population size, the response time of the GA can be improved. However, there is a trade-off between the selected route and the response time.

The GA tries to find a route with a good fitness value, but based on genetic operators probability this may be not the global optimum. In the case of mobile Ad-hoc networks, it is better to find a route very fast in order to have a good response time to the speed of topology change, than to search for the best route but without meaning, because the network topology is changed and this route does not exist any-more.

In this paper, we use GAs and multi-objective optimization for QoS routing in Ad-hoc Networks. In order to reduce the search space of GA, we implemented a Search Space Reduction Algorithm (SSRA), which reduces the search space of GAMAN. We evaluate the performance of GAMAN by computer simulations and show that GAMAN has better behaviour than a previous method called Genetic Load Balancing Routing (GLBR).

The paper is organized as follows. In Section 2, we discuss QoS Support in Ad-hoc Networks and QoS metrics. In Section 3, we deal with related work on QoS routing. In Section 4, we present GA-based QoS routing. Our proposed multi-objective optimization method is presented in Section 5. The simulation results are presented in Section 6. Finally, conclusions are given in Section 7. 


\section{QoS support in Ad-hoc networks and QoS metrics}

\subsection{QoS support in Ad-hoc networks: Issues and difficulties}

The QoS is usually defined as a set of services requirements that needs to be met by the network while transporting a packet stream from a source to a destination. The network needs are governed by the service requirements specified by the end user applications. The network is expected to guarantee a set of measurable pre-specified service attributes to the users in terms of end-to-end performance metrics, such as delay, bandwidth, transmission success ratio, packet loss, and jitter. The power consumption and service coverage area are two other QoS metrics that are more specific to Ad-hoc networks [16].

The Ad-hoc networks differ from traditional wire-line networks. The difference introduce unique issues and difficulties for supporting QoS in Ad-hoc networks environment, which are summarized in following.

\section{- Unpredictable Link Properties}

Wireless media is very unpredictable. Packet collision is intrinsic to wireless network. Signal propagation faces difficulties such as signal fading, interference, and multi-path cancellation. All these properties make the measure of bandwidth and delay of a wireless link unpredictable.

\section{- Hidden-Exposed Terminal Problem}

Multi-hop packet relaying introduces the hidden-exposed terminal problem. This problem happens when signals of two nodes which are out of transmission range of each other collide at the common receiver. Because of the local nature of transmissions, hidden and exposed stations abound in Ad-hoc networks. A hidden station is a host that is within the range of the receiver but not the transmitter, while an exposed station is within the range of the transmitter but not the receiver.

- Node Mobility

Mobility of the nodes create a dynamic network topology. Links will be dynamically formed when two nodes come into transmission range of each other and be torn down when they move out of the range.

\section{- Route Maintenance}

The dynamic nature of the network topology and the changing behaviour of the communication medium make the precise maintenance of network information very difficult. Thus, the routing algorithms in Ad-hoc networks have to operate with inherently imprecise information. Furthermore, in Ad-hoc networks environments, node can join and leave any-time. The established routing paths may be broken even during the process of data transfer. Thus arises the need for maintenance and reconstruction of routing paths with minimal overhead and delay.

\section{- Limited Battery Life}

Mobile devices generally are dependent on finite battery sources. The resource allocation for QoS provisioning must consider the residual battery power and the rate of battery consumption corresponding to the resource utilization. Thus, all the techniques for QoS provisioning should be power-aware and power-efficient.

\section{- Security}

Security can be considered as a QoS attribute. Without adequate security, unauthorized access and usages may violate the QoS negotiations. The nature of broadcasts in wireless networks potentially results in more security exposures. The physical medium of communications is inherently insecure. So, we need to design security-aware routing algorithms for Ad-hoc networks. 


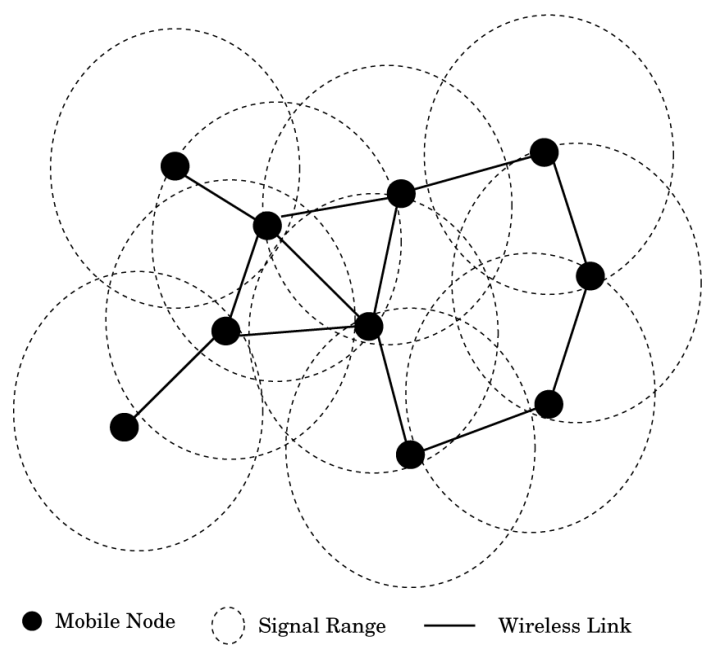

Fig. 1. An Ad-hoc network.

\subsection{QoS routing metrics}

In [17], the authors present the following metrics for QoS route discovery and selection.

- Minimum Required Throughput or Capacity (bps)

This is the desired application data throughput.

- Maximum Tolerable Delay (s)

Usually defined as the maximum tolerable end-to-end (source to destination) delay for data packets.

- Maximum Tolerable Delay Jitter

One widely accepted definition of this metric is the difference between the upper bound on end-toend delay and the absolute minimum delay. The former incorporates the queuing delay at each node and the latter is determined by the propagation delay and the transmission time of a packet. This metric can also be expressed as delay variance.

- Maximum Tolerable Packet Loss Ratio (PLR)

The acceptable percentage of total packets sent, which are not received by the transport or higher layer agent at the packet's final destination node.

An application may typically request a particular quality of service by specifying its requirements in terms of one or more of the above metrics.

\section{Related work on QoS routing}

In this section, we will review the routing schemes that can support QoS in Ad-hoc networks. In Fig. 1 is shown an Ad-hoc network. The wireless topology derived from Fig. 1 is shown in Fig. 2. The mobile nodes are labelled as A, B, C, .., K. The numbers beside each edge represent the available bandwidths of the wireless links. Suppose we want to find a route from Source Node (SN) A to a Destination Node (DN) G. For conventional routing using shortest path (in terms of the number of hops) as metric, the route "A-B-H-G" will be chosen. It is quite different in QoS route selection. Suppose we consider bandwidth as QoS metric and desire to find a route from A to $G$ with a minimum bandwidth of 4 . Now, the feasible 


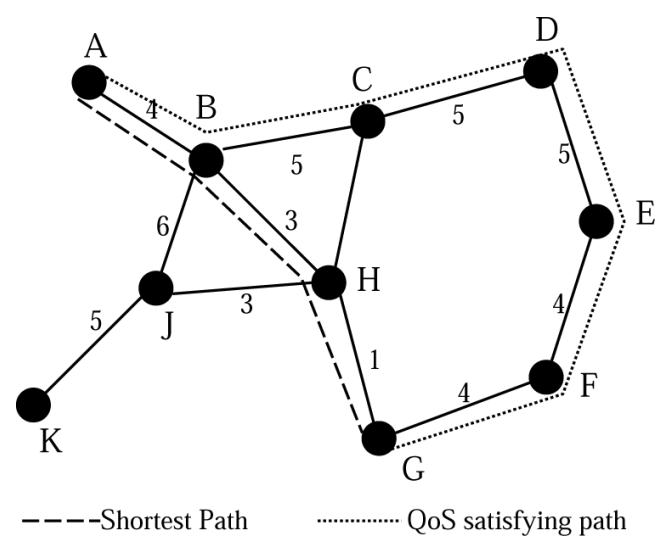

Fig. 2. An example of QoS in Ad-hoc networks.

route will be "A-B-C-D-E-F-G". The shortest path route "A-B-H-G" will not be adequate for providing the required bandwidth.

The primary goal of the QoS routing protocol is to determine a path from a SN to a DN that satisfies the needs of the desired QoS. The QoS path is determined within the constraints of minimal search, distance and traffic conditions. We discuss some QoS routing algorithms in following [17].

\subsection{Ticket-based probing algorithm}

A ticket-based probing algorithm with imprecise model was proposed by Chen and Nahrstedt [9]. While discovering a QoS-aware routing path, this algorithm tries to limit the amount of flooding (routing) messages by issuing a certain amount of logical tickets. Each probing message must contain at last on ticket. When a probing message arrives at a node, it may be split into multiple probes and forwarded to different next-hops. Each child probe will contain a subset of tickets from their parent. Obviously, a probe with a single ticket cannot be split any more. When one or more probe(s) arrive(s) at the destination, the hop-by-hop path is known and delay/bandwidth information can be used to perform resource reservation for the QoS-satisfying path.

In wire-line networks, a probability distribution can be calculated for a path based on the delay and bandwidth information. In a mobile Ad-hoc network, however, building such a probability distribution is not suitable, because wireless links are subject to breakage and state information is imprecise in nature. Hence a simple imprecise model was proposed for ticket-based probing algorithm. It uses history and current (estimated) delay variations and a smoothing formula to calculate the current delay, which is represented as a range of [delay $-\delta$, delay $+\delta$ ]. To adapt to the dynamic topology of mobile Ad-hoc networks, this algorithm allows different level of route redundancy. It also uses re-routing and pathrepairing techniques for route maintenance. When a node detects a broken path, it will notify the SN, which will reroute the connection to a new feasible path, and notify the intermediate nodes along the old path to release the corresponding resources. Unlike the re-routing technique, path-repairing technique does not find a completely new path. Instead, it tries to repair the path using local reconstructions.

\subsection{CEDAR}

CEDAR, a Core Extraction Distributed Ad-hoc Routing is proposed as a QoS routing scheme for small and medium scale mobile Ad-hoc networks [10]. It dynamically establishes the core of the network, and 
then incrementally propagates the link states of stable high-bandwidth links to the core nodes. The route computation is on-demand basis, and is performed by the core nodes using only local state. CEDAR has three key components has follows.

Core Extraction: A set of nodes elected to form the core that maintains the local topology of the nodes in its domain, and also perform route computation. The core nodes are elected by approximating a minimum dominating set of the mobile Ad-hoc Networks.

Link State Propagation: QoS routing in CEDAR is achieved by propagating the bandwidth availability information of stable links to all core nodes. The basic idea is that the information about stable high-bandwidth links can be made known to nodes far away in the network, while information about the dynamic or low bandwidth links remains within the local area.

Route Computation: Route computation first establishes a core path from domain of the SN to the domain of DN. Using the directional information provided by core path, CEDAR iteratively tries to find a partial route from the source to the domain of the furthest possible node in the core path satisfying the requested bandwidth. This node then becomes the source of the next iteration. In CEDAR approach, the core provides an efficient and low-overhead infrastructure to perform routing, while the state propagation mechanism ensures the availability of the link-state information at the core nodes without incurring high overheads.

\subsection{Interference-aware QoS routing}

In [18], the authors consider throughput-constrained QoS routing based on knowledge of the interference between links. So-called clique graphs are established, which reflect which links interfere with each other, thereby preventing simultaneous transmission. The proposed solution operates by first recording the channel usage (bps) of each existing data session on each link. It is noted that the total channel usage of the sessions occupying the links within the same clique must not exceed the channel capacity. A link's residual capacity is then calculated by subtracting the channel usage of all sessions on links in the same clique from the link's nominal capacity. This link capacity information may then be used in any known distributed Ad-hoc routing protocol to solve the throughput-constrained routing problem.

\subsection{Cross-layer multi-constraint QoS routing}

In [19], the author focuses on performing multi-constraint QoS routing with three metrics: delay, link reliability and throughput. The author reiterates the fact that the multi-constraint QoS routing problem is NP-complete when a combination of additive and multiplicative metrics is considered. Among the above metrics, delay is additive, link reliability is multiplicative and achievable throughput is concave. However, methods have been proposed for reducing this NP-complete problem to one that can be solved in polynomial time. In one such method, all QoS metrics, except one, take bounded integer values. Then, the task of finding a path to satisfy all constraints can be performed by a modified Dijkstra's algorithm.

In [19], the multiplicative metric is reduced to an additive one by taking the logarithm of the reliability percentage of a link. Also, the delay metric is reduced such that each link is represented by the percentage of the allowable total delay it introduces. The resulting problem in the new metric space can be solved in polynomial time. Then, a modified Bellman-Ford or Dijkstra's algorithm with the new reliability metric for link weights can be used to find an approximation to the optimal path.

An obvious advantage of this approach is the concurrent consideration of several important QoS metrics in path selection. However, the QoS state for all paths must be discovered and kept fresh. This incurs extra overhead and the details of this mechanism are not discussed in the paper. 


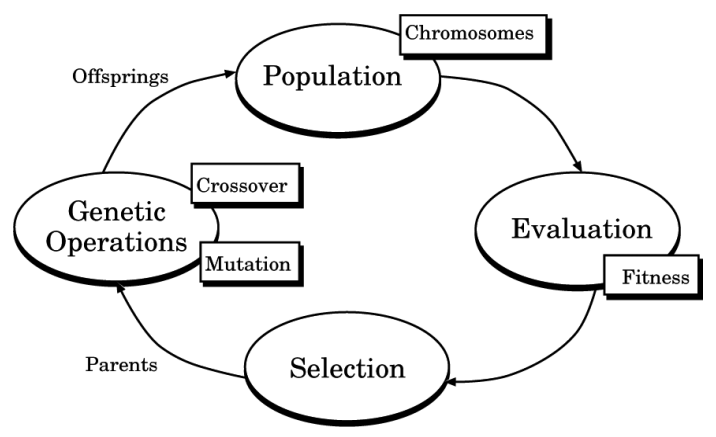

Fig. 3. GA cycle.

\section{GA-based QoS routing}

\subsection{GA cycle}

The GA cycle is shown in Fig. 3. At the beginning, an initial population of potential solutions is created as a starting point for the search. In the next stage, the performance (fitness) of each individual is evaluated with respect to the constraints imposed by the problem. Based on each individual's fitness, a selection mechanism chooses "parents" for the crossover and mutation operators.

The crossover operator takes two chromosomes and swaps part of their genetic information to produce new chromosomes. The mutation operator introduces new genetic structures in the population by randomly modifying some of genes, helping the search algorithm to escape from local optimum.

The offspring produced by the genetic manipulation process are the next population to be evaluated. GA can replace either a whole population or just its less fit members. The creation-evaluation-selectionmanipulation cycle repeats until a satisfactory solution to the problem is found, or some other termination criteria are met [20].

\subsection{GAMAN QoS routing algorithm}

In [21], we proposed a GA-based QoS routing algorithm for mobile Ad-hoc networks. We will explain in following GAMAN algorithm.

\subsubsection{Network model assumptions}

We assume that all the hosts communicate on the same shared wireless channel. Each node has a unique identifier and has at least one transmitter and one receiver. Assume that the effective transmission distance of every node is equal. Two nodes are neighbours and have a link between them if they are in the transmission range of each other.

We assume that there exists a neighbour discovering protocol. Each node periodically transmits a BEACON packet identifying itself, so that every node knows the set of neighbour nodes. We assume the existence of a MAC protocol, which resolves the media contention, supports resource reservation and ensures that among the neighbours in the local broadcast range only the intended receiver keeps the message and the other neighbours discard the message.

We assume small to medium networks. For larger networks some cluster based algorithms or distributed algorithms can be used. We assume that the change in network topology is frequent, but not frequent enough to render any sort of route computation useless. Specifically, we assume that topology changes 
are typically followed by at least a short period of stability. Note that we only care about the relative mobility of hosts not the absolute mobility of the hosts.

In particular, even if a platoon of solders or cars is moving, the mobile Ad-hoc Networks would be considered to be stable as long as the neighbourhood of each solder or car does not change. The links between the stationary or slow moving nodes are likely to exist continuously. Such links are considered as stationary links. The links between fast moving nodes are likely to exist only for a short period of time. Such links are considered as transient links. A routing path should use the stationary links whenever possible in order to reduce the probability of the path breaking when the network topology changes.

\subsubsection{GAMAN goals}

We consider a type of mobile Ad-hoc network whose topologies are not changing that fast to make the QoS routing meaningless. We want to emphasize that GAMAN supports soft QoS without hard guarantees. The soft QoS means that there may exist transient time periods when the required QoS is not guaranteed due path breaking or network partition. However, the required QoS should be ensured when the established paths remain unbroken. Many multimedia applications accept soft QoS and use adaptation techniques to reduce the level of QoS disruption.

In CEDAR, the bandwidth is used as the only QoS parameter for routing. Also, in ticked-based routing method the delay and bandwidth are used for QoS routing but not together. They are implemented as different algorithms. In this version, the GAMAN algorithm uses two parameters: delay and transmission success rate to decide the QoS path. To our best knowledge, this is the first work for MANET QoS routing to consider two QoS parameters.

The routing in mobile Ad-hoc networks has the following goals: a) the routing computation for small networks should be made at the SN in order to avoid the computation at intermediate nodes; $b$ ) as few nodes as possible must be involved in route computation; c) each node must only care about the routes corresponding to its destination, and must not be involved in frequent topology updates for parts of the network to which it has no traffic; d) broadcast must be avoided as far as possible; e) it is desirable to have a backup route when the primary route has become stale and is being recomputed; f) messages should be not cycled around loops.

Our GAMAN algorithm can satisfy these requirements: a) the GAMAN is a source-based routing algorithm; b) by using a SSRA and small population size few nodes are involved in route computation; c) by taking a sub-population, the nodes in this sub-population care only about the routes in this subpopulation; d) the broadcast is avoided because the information is transmitted only for the nodes in a population; e) the GA search different routes and they are sorted by ranking them. So the first one is the best route, but other ranked routes can be used as backup routes; f) by using a tree based GA method, the loops can be avoided.

In summary, our goal is to compute good routes quickly, and react to the dynamics of the network very fast. As a results, we sacrifice optimality of routes. Robustness, rather than optimality is the key requirement.

\subsubsection{SSRA}

By SSRA a network with many nodes will be reduced in a network with a small number of nodes. Thus, GAMAN is able to cope with increase of number of nodes in Ad-hoc networks.

The flowchart of SSRA is shown in Fig. 4. The key element of SSRA is topology extraction. In order to extract the topology, the node information and QoS requirement are needed. In this work, we use TSR (Th), DT (Th), CC (Th) to specify the QoS requirements. If DT $<$ DT (Th), TSR $>$ TSR (Th), CC 


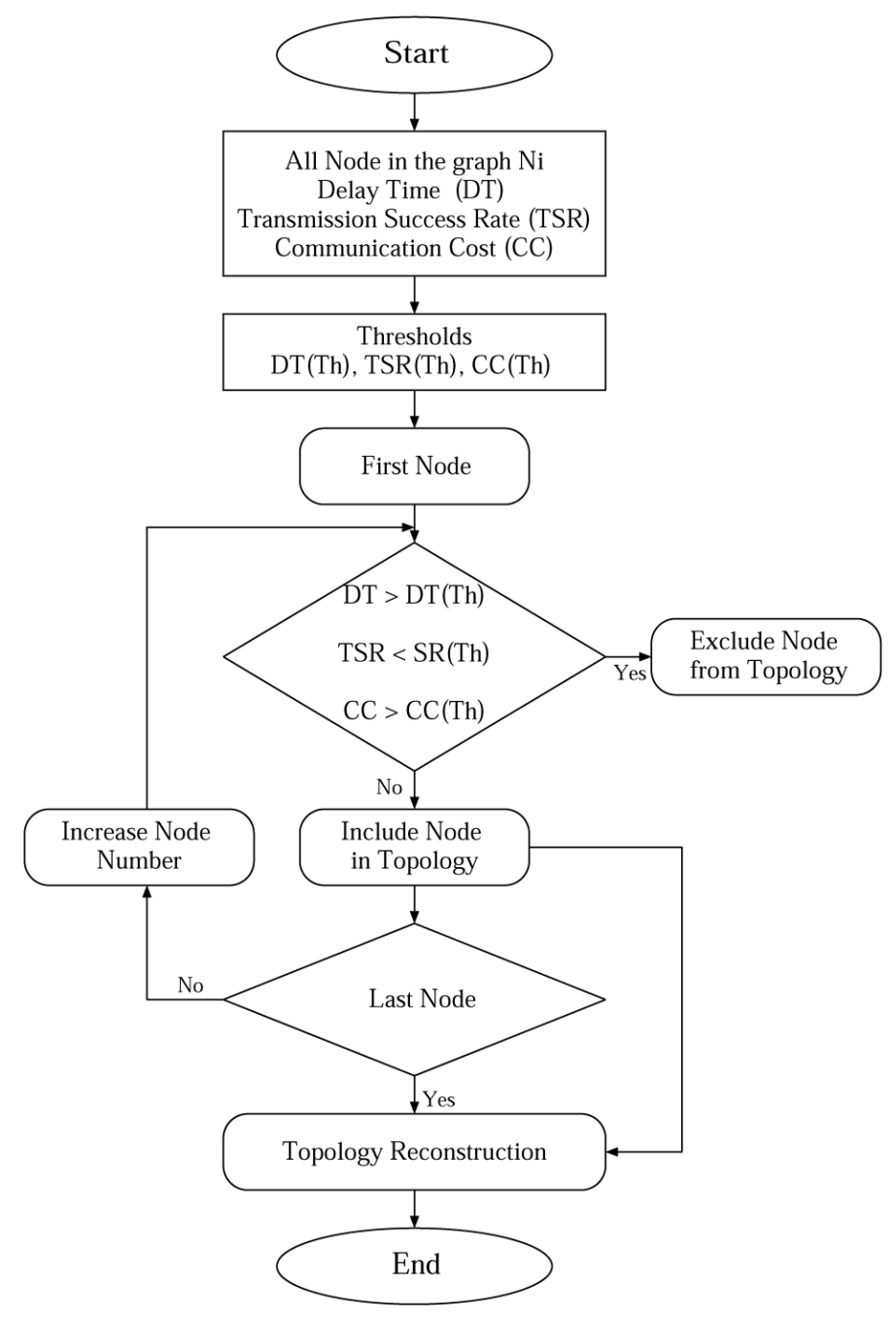

Fig. 4. SSRA Flowchart.

$<\mathrm{CC}$ (Th), then the node is included in the extracted topology, otherwise the node is excluded from the extracted topology. The procedure is repeated until all nodes are finished. After all nodes are checked, the network topology is constructed and the complete procedure is finished.

\subsubsection{Gene coding}

To explain this procedure, we use a small mobile Ad-hoc network with 8 nodes as shown in Fig. 5. Let consider node $\mathrm{A}$ as a SN and node $\mathrm{H}$ as a DN. All routes are expressed by the network tree model shown in Fig. 6. The shaded areas show the same routes from node $\mathrm{C}$ to $\mathrm{H}$. In order to decrease the chromosome gene number, the network tree model of Fig. 6 is reduced as shown in Fig. 7. In the reduced network tree model, each tree junction is considered as a gene and the path is represented by the chromosome.

The most important factor to achieve efficient genetic operations is gene coding, because it has influence on the efficiency of genetic operations.

In [22], the GLBR method which uses GA has been proposed. The adaptive routing mechanism has a load balancing system among alternative paths. In the GLBR method, the genes are put in a chromosome 


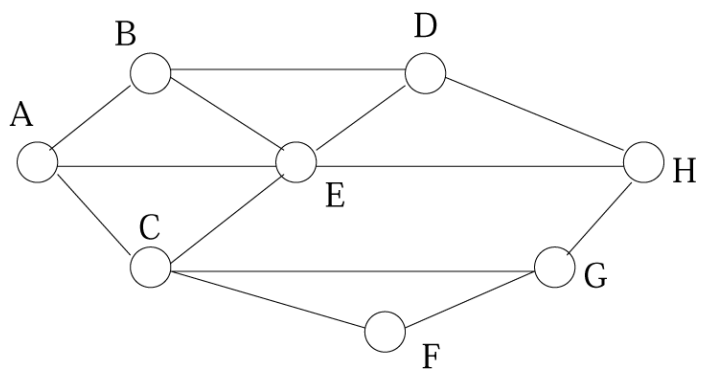

Fig. 5. A mobile Ad-hoc network with 8 nodes.

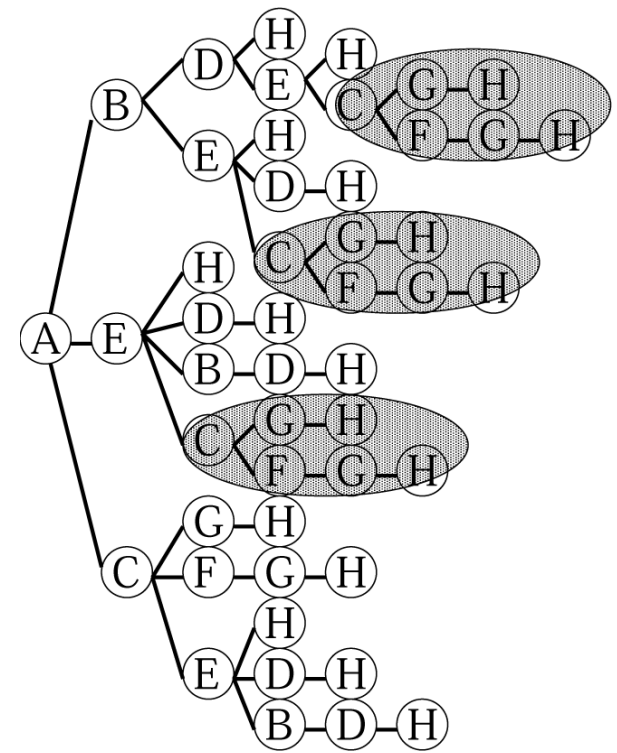

Fig. 6. Network tree model.

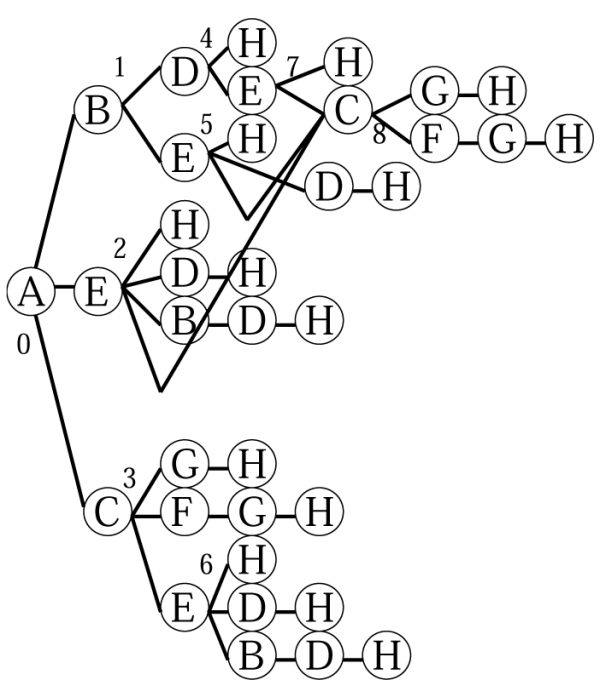

Fig. 7. Reduced network tree model.

in the same order the nodes form a route as shown in Fig. 8(a). Therefore, the chromosomes have different sizes. If genetic operations are chosen randomly, the new off-springs of a population may be unsuitable individual populations. Also, because the individuals of a population have different sizes, the crossover operations are complicated.

In order to simplify the genetic operations of GLBR method, in GAMAN, the network is changed in a tree, then the tree network is reduced by grouping together the same routes. After, the tree reduction, the tree junctions are expressed by genes as shown in Fig. 8(b). In Fig. 8(b) is shown a chromosome example for the route "A-B-D-E-C-F-G-H" of network example. Each chromosome expresses only one route. The genes contain the information of the adjacent nodes. As is shown in Fig. 8(b), the chromosomes have the same length. Therefore, the crossover operation becomes very easy. The genes in a chromosome have two states "active" and "inactive". A gene is called active if the junction is in the route, otherwise the gene is in "inactive" state.

In GLBR method, the interaction between the adjacent genes in a chromosome is necessary. While, in GAMAN this interaction is not necessary. So, the mutation operation also becomes easy. Furthermore, the GAMAN has a good gene coding method, which is able to create various individuals, which result in a fast evolution. 


\begin{tabular}{|l|l|l|l|l|l|l|l|}
\hline 0 & 1 & 2 & 3 & 4 & 5 & 6 & 7 \\
\hline A & B & D & E & C & F & G & H \\
\hline
\end{tabular}

(a) GLBR gene coding.

\begin{tabular}{|c|c|c|c|c|c|ccc|c|}
\multicolumn{1}{c}{0} & 1 & 2 & 3 & 4 & 5 & 6 & 7 & 8 \\
\hline BEC & DE & $\begin{array}{r}\text { HD } \\
\text { BC }\end{array}$ & GFE & HE & HDC & HDB & HC & GF \\
\hline
\end{tabular}

(b) GAMAN gene coding.

Fig. 8. Chromosome examples for the route "A-B-D-E-C-F-G-H".

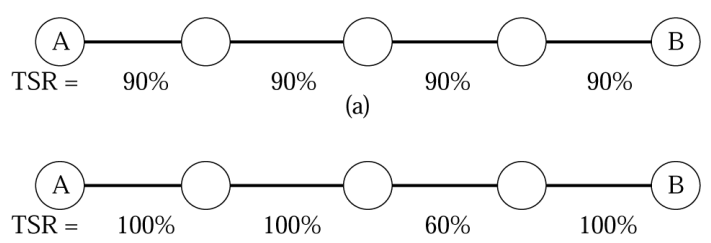

(b)

Fig. 9. An example of TSR calculation.

In GAMAN, the selection operation uses both the ranking and elitist models. As the crossover method is used the single point crossover. In the mutation operation, the genes are chosen randomly in the range from zero up to mutation probability $p_{-}$mutation $\leqslant \frac{1}{\ell}$, where $l$ is the chromosome length.

\subsubsection{QoS routing parameters}

The GAMAN algorithm can use many parameters for QoS routing. In following we consider the case for two QoS parameters: Delay Time (DT) and Transmission Success Rate (TSR). The DT means the time it takes a packet to go from one node to another one. The TSR shows the rate of correctly transmitted packets (without loss).

Let consider a wireless network with wireless nodes and links as shown in Fig. 9. The node A is the source node and node B is the destination node. Let node A sends to node B 10 packets. The total TSR value for Figs 9(a) and 9(b) is calculated by Eqs (1) and (2), respectively.

$$
\begin{aligned}
& 10 \times 0.9 \times 0.9 \times 0.9 \times 0.9=6.561 \\
& 10 \times 1.0 \times 1.0 \times 0.6 \times 1.0=6.000
\end{aligned}
$$

The best route in this case is that of Fig. 8(a), because the total TSR is higher compared with that of Fig. 8(b).

Let consider another example, when the values of DT and TSR are considered as shown in Fig. 10. The value of $\mathrm{T}$ parameter is decided as follows.

$$
T=\frac{\sum_{i=1}^{n} D T_{i}}{\prod_{i=1}^{n} T S R_{i}}
$$

where $n$ is the number of wireless links in a path. 


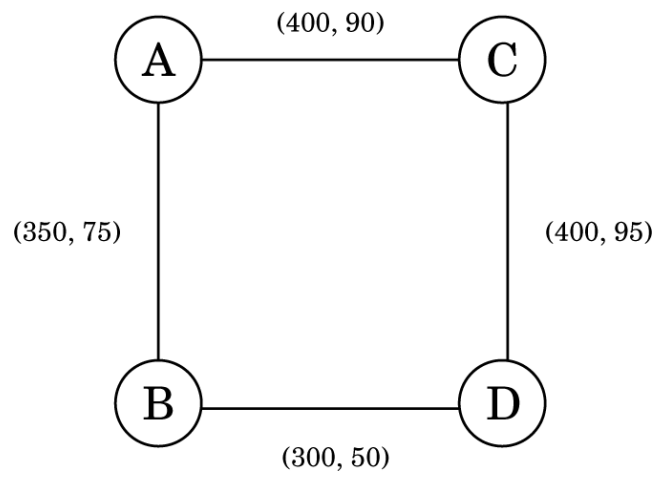

Fig. 10. A network example for mobile Ad-hoc network QoS routing.

When node A wants to communicate with node D, there are two possible routes: "A-B-D" and "A-C-D". The T value for these routes is calculated by Eqs (4) and (5), respectively.

$$
\begin{aligned}
& T_{A-B-D}=\frac{350+300}{75 \times 50}=\frac{650}{3750}=0.1733 \\
& T_{A-C-D}=\frac{400+400}{90 \times 95}=\frac{800}{8550}=0.0468
\end{aligned}
$$

The delay time of "A-B-D" route is lower than "A-C-D" route, but the T value of "A-C-D" route is lower than "A-B-D", so "A-C-D" route is the better one. This shows that other good candidate routes can be found when two QoS parameters are used for routing in mobile Ad-hoc networks.

\subsubsection{GAMAN operation}

The GAMAN is a source-based routing mechanism and uses two QoS parameters for routing. When a node of mobile Ad-hoc network wants to transmit information to a DN, this node becomes the SN. The network first is transformed in a tree network with the SN as the root of tree. After that, the tree network is reduced in the parts where are the same routes. By reducing the tree network, the chromosome length is shorten so the genetic operations become simple.

After the reduction of the tree network, the tree junctions are coded as genes. The genes in a chromosome have the information of the adjacent nodes. Because, the individual and chromosome are the same, the route is represented by the chromosome and the population is a collection of wireless links.

After the gene coding, GAMAN starts the genetic operations. First, an initial population is selected. In the selected population, the ranking selection model is used to select individuals in order to carry out the genetic operations. The ranking model ranks each individual by their fitness. The rank is decided based on the fitness and the probability is decided based on the rank. The individual fitness is based on $\mathrm{T}$ value. When $\mathrm{T}$ value is small, the individual fitness is high.

The genetic operations are the crossover and mutation. The GAMAN algorithm uses the single point crossover, because simple operations are needed to get a fast response. In the mutation operation, the genes are chosen randomly in the range from zero up to mutation probability $p_{-}$mutation $\leqslant \frac{1}{\ell}$, where $l$ is the chromosome length.

After the crossover and mutation, the elitist model is used. Based on the elitist model the individual which has the highest fitness value in a population is left intact in the next generation. Therefore, the best value is always kept and the routing algorithm can converge fast. The off-springs produced by the 


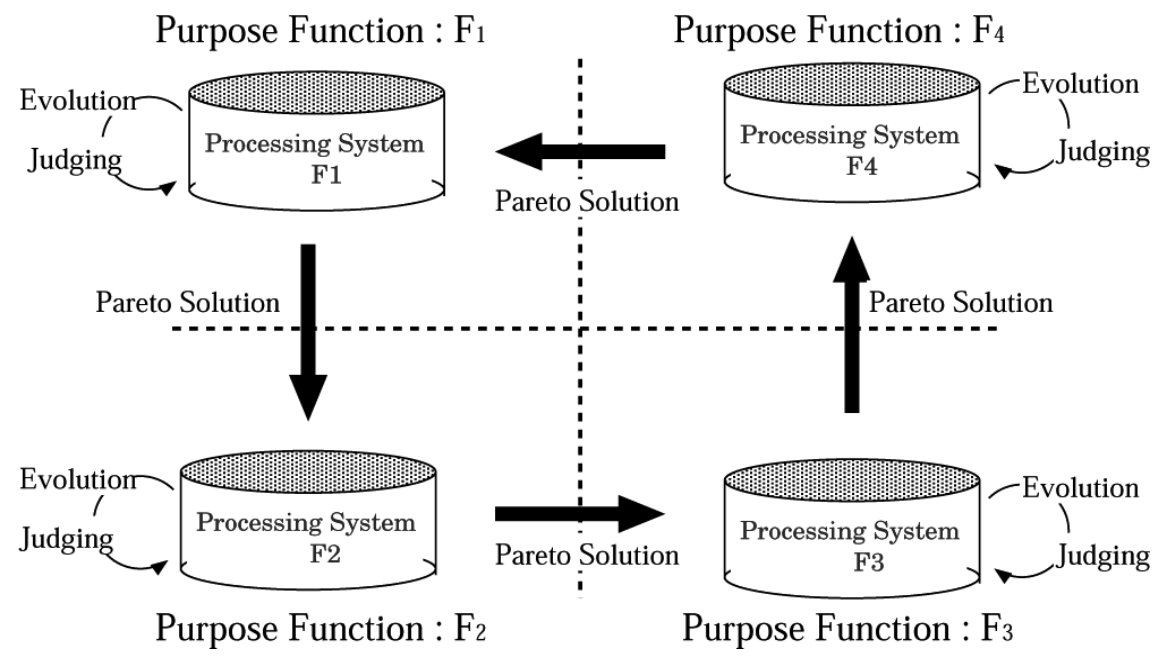

Fig. 11. Multiple-objective optimization.

genetic operations are the next population to be evaluated. The genetic operations are repeated until the initialized generation size is achieved or a route with a minimum $\mathrm{T}$ value is found.

The route selection in GAMAN is based on T value, which is the ratio of DT with TSR. T is used as a fitness function to evaluate the selected individuals (routes). By minimizing the $T$ value, the DT value is minimized and the TSR value is maximized. This means that a packet from node SN to node DN is transmitted with a small delay and a high transmission success rate.

\section{Proposed multi-objective optimization method}

In this section, we propose a new method for Ad-hoc networks considering GA and multi-objective optimization. The proposed method uses the multi-division group model for multi-objective optimization. When a function can be divided in different objective functions, the global domain can be divided in different domains and each individual can evolve in its domain.

The procedure is shown in Fig. 11, where four different objective functions are independent from each other and each process is operating independently in each domain. In Fig. 12 is shown an example of Delay Time (DT) and Communication Cost (CC). The vertical axis shows the DT and horizontal axis the CC. The DT and CC have trade-off relations. The points in the figure show the individuals (routes). The individuals which are in the left-upper part of the figure have the lowest CC. On the other hand, the individuals which are in the right-lower part of the figure have the lowest DT values. The individuals which are in the shaded area have good values for both DT and CC. The shaded area is called "pareto solution". The individuals near pareto solution can be found by exchange the solutions of different domains.

\subsection{QoS routing search engine}

The structure of QoS Routing Search Engine (QRSE) is shown in Fig. 13. It includes Cache Search Engine (CSE) and GAMAN. CSE and GAMAN operate independently, but they cooperate together to update the route information. When the RSE receives a request for QoS routing from a client, it forwards 


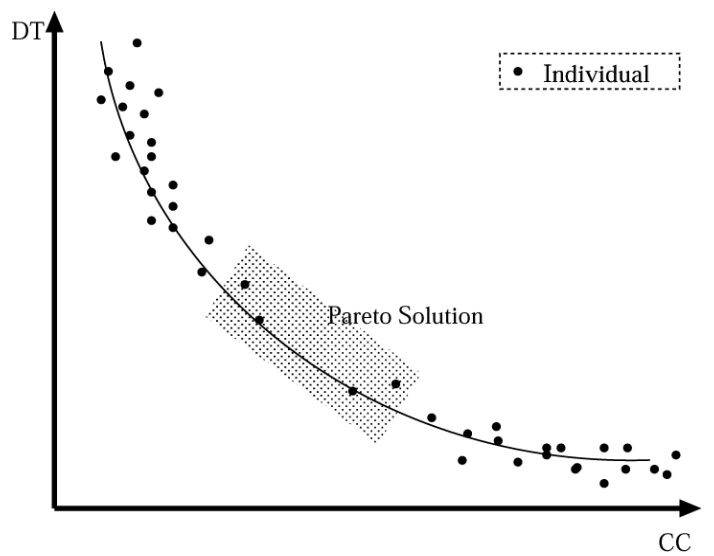

Fig. 12. Pareto solution for DT and CC.

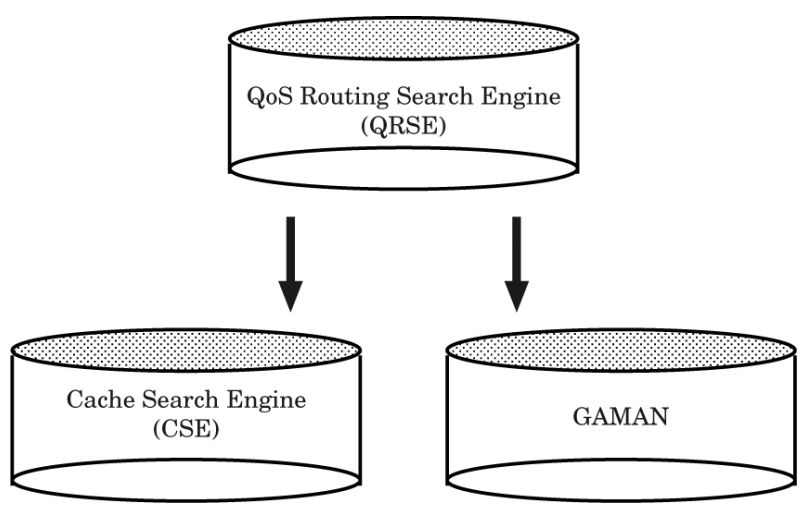

Fig. 13. RSE structure.

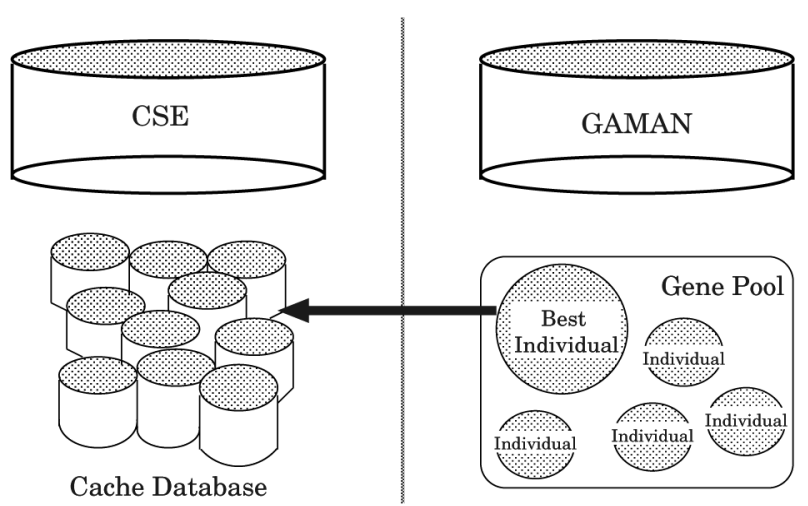

Fig. 14. CSE and GAMAN cooperation1.

the request in parallel to CSE and GAMAN. Then, the CSE and GAMAN search in parallel to find a route satisfying the required QoS. The CSE searches for a route in the cache database (in the cache database, the destination and route information is saved as a database item). If the found route by CSE 


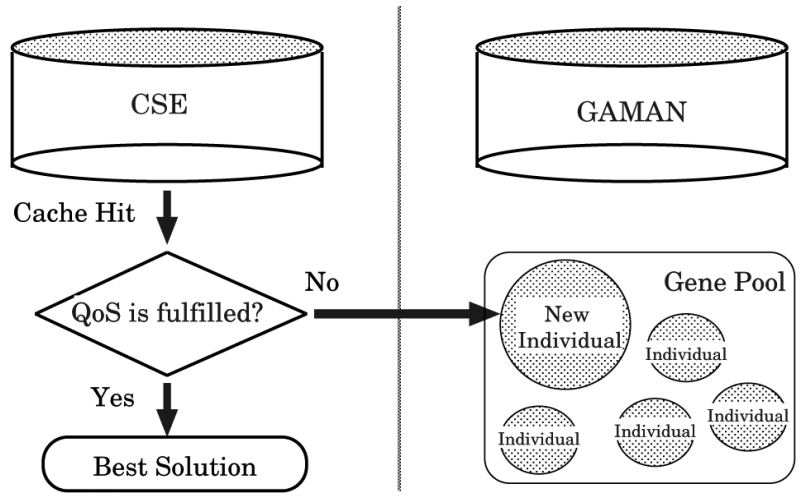

Fig. 15. CSE and GAMAN cooperation2.

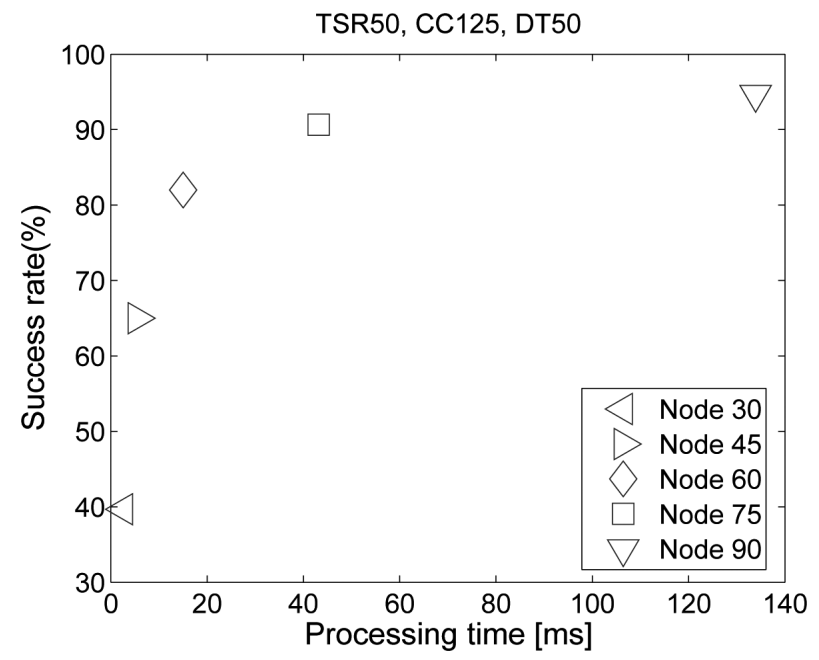

Fig. 16. Algorithm success rate versus processing time.

satisfies the required QoS, this route information is sent to RSE, otherwise the route is put in the gene pool as new individual. If a QoS route can not be found by CSE, the route found by GAMAN is sent to RSE.

\subsection{CSE-GAMAN cooperation and database updating}

The database information should be updated because the network traffic and the network state change dynamically. In order to update the database, the CSE and GAMAN cooperate together. When the GAMAN finds a good QoS routing, it puts this route information in the cache database as shown in Fig. 14. The route information which is used frequently is given high priority, thus this route can be searched very fast by CSE. In the case when the CSE finds a route in the database, but this route does not satisfy the required QoS by client, this route information is put as a new individual in the genepool of GAMAN as shown in Fig. 15. 


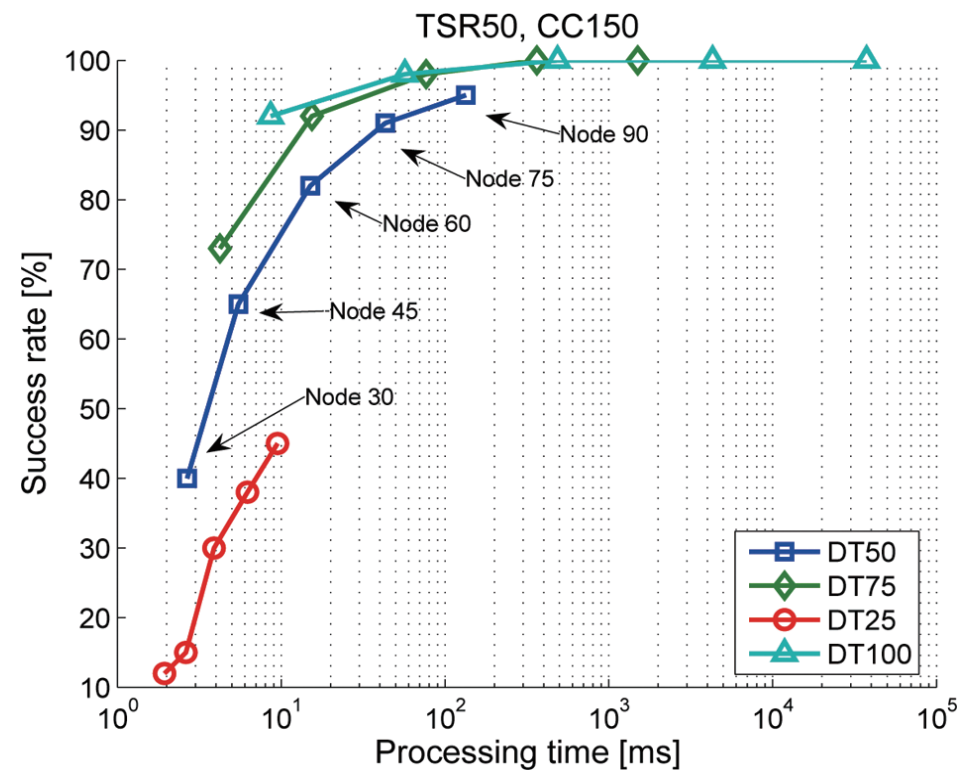

Fig. 17. Algorithm success rate versus processing time for different DTs.

\section{Simulation results}

We show in Figs 16 and 17 the simulation results for SSRA. By reducing the search space, the GAMAN can find faster a new route. We use as QoS parameters: Transmission Success Rate (TSR), DT and CC. As the simulation environment was used desk-top computer (OS: Windows XP Pro; IDE: Visual C++6.0; CPU: Athlon64 +3500, Memory: $1024 \mathrm{MB}$ ). The QoS parameters were set up in a random way. The DT for each link was set between 1 and 100, TSR was set between 1 and $100 \%$ and CC between 1 and 200 units. In Fig. 16, we set the threshold parameters $T S R(T h)=50, C C(T h)=125$, $D T(T h)=50$. Then, we changed the number of nodes from 30 to 45, 60, 75 and 90 and measure the algorithm success rate. As shown in these figures, with the increase of the number of nodes the success rate to find a feasible route increases. In Fig. 17, we consider $T S R(T h)=50, C C(T h)=150$ and change $D T(T h)$ from 25 to 100 . As shown in the figure with the increase of the DT threshold the success rate is increased. However, when the threshold is very small the success rate is less than $20 \%$. Therefore, there are trade-offs for deciding the threshold parameters.

In Table 1, we show the simulation results of the proposed method for population size 10 and for different number of generations, crossover rates and mutation rates.

We can see that with increase of mutation rate, the average number of generations and average processing time is decreased. But, with increase of crossover rate, the average number of generations and average processing time is increased. However, when the mutation rate is $8 \%$, the performance of proposed method is better than $10 \%$. Also for crossover rate $70 \%$ and mutation rate $8 \%$ we have the best performance. In Table 2 are shown the simulation results of GLRB. Comparing Tables 1 and 2, GAMAN has a better performance than GLBR.

The simulation results of GAMAN for the time needed for one generation are shown in Table 3. If there are few individuals in the population, the Generation Number (GN) which shows the number of generations needed to find a solution becomes large. On the other hand, when the number of individuals is high, the GN to find a solution becomes small. However, when the number of individuals is 12 and 
Table 1

GAMAN Simulation results for population size 10

\begin{tabular}{cccc}
\hline $\begin{array}{c}\text { Mutation } \\
\text { rate [\%] }\end{array}$ & $\begin{array}{c}\text { Crossover } \\
\text { rate [\%] }\end{array}$ & $\begin{array}{c}\text { Average no. } \\
\text { of generations }\end{array}$ & $\begin{array}{c}\text { Average processing } \\
\text { time [ms] }\end{array}$ \\
\hline 1 & 70 & 15.7 & 468.2 \\
& 80 & 16.7 & 487.5 \\
& 90 & 21.2 & 590.7 \\
5 & 100 & 19.3 & 536.7 \\
& 70 & 11.3 & 213.3 \\
& 80 & 9.8 & 185.9 \\
& 90 & 10.7 & 203.9 \\
8 & 100 & 14.1 & 275.2 \\
& 70 & 7.4 & 120.6 \\
& 80 & 7.8 & 137.5 \\
10 & 90 & 7.9 & 133.3 \\
& 100 & 9.1 & 157.3 \\
& 70 & 9.1 & 149.3 \\
& 80 & 8.9 & 143.8 \\
& 90 & 8.2 & 135.5 \\
& 100 & 7.4 & 120.6 \\
\hline
\end{tabular}

Table 2

GLBR Simulation results for population size 10

\begin{tabular}{cccc}
\hline $\begin{array}{c}\text { Mutation } \\
\text { rate [\%] }\end{array}$ & $\begin{array}{c}\text { Crossover } \\
\text { rate [\%] }\end{array}$ & $\begin{array}{c}\text { Average no. } \\
\text { of generations }\end{array}$ & $\begin{array}{c}\text { Average processing } \\
\text { time [ms] }\end{array}$ \\
\hline 1 & 70 & 20.7 & 2017.1 \\
& 80 & 23.6 & 1652.8 \\
& 90 & 22.3 & 1842.9 \\
5 & 100 & 21.5 & 1872.0 \\
& 70 & 15.4 & 497.3 \\
& 80 & 17.5 & 647.4 \\
& 90 & 17.7 & 668.0 \\
8 & 100 & 22.1 & 589.9 \\
& 70 & 12.4 & 404.6 \\
& 80 & 10.0 & 389.9 \\
10 & 90 & 24.4 & 538.8 \\
& 100 & 21.4 & 494.4 \\
& 70 & 14.2 & 384.1 \\
& 80 & 11.5 & 442.1 \\
& 90 & 17.2 & 468.2 \\
& 100 & 17.3 & 368.8 \\
\hline
\end{tabular}

16, the difference is very small. This is because some individuals become the same in the genepool. Considering the exchange of individuals between domains, it can be seen that when the exchange interval is short the solution can be found very fast. This shows that by exchanging the individuals the algorithm can approach very quickly to the pareto solution.

In Fig. 18 are shown the simulation results for number of generations and the processing time. In Fig. 18(a), we show the Generation Size (GS) versus the number of individuals. While, in Fig. 18(b) is shown the processing time versus the number of individuals. We consider 4 cases when the number of exchanging individuals is $3,5,7$, and 10 , respectively. If there are few individuals in the population, the GS which shows the number of generations needed to find a solution becomes large. 
Table 3

Time needed for one generation (ms)

\begin{tabular}{ccccc}
\hline Number of & \multicolumn{4}{c}{ GN exchange } \\
\cline { 2 - 5 } individuals & 3 & 5 & 7 & 10 \\
\hline 4 & 44.43 & 50.45 & 46.19 & 55.59 \\
8 & 26.83 & 28.01 & 40.26 & 31.17 \\
12 & 23.55 & 26.49 & 26.04 & 26.71 \\
16 & 22.22 & 22.23 & 23.25 & 24.04 \\
\hline
\end{tabular}

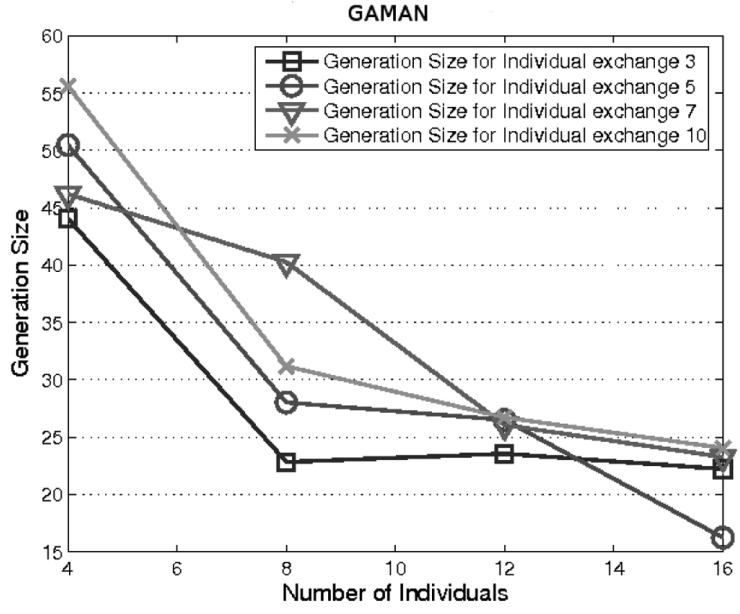

(a) Number of generations

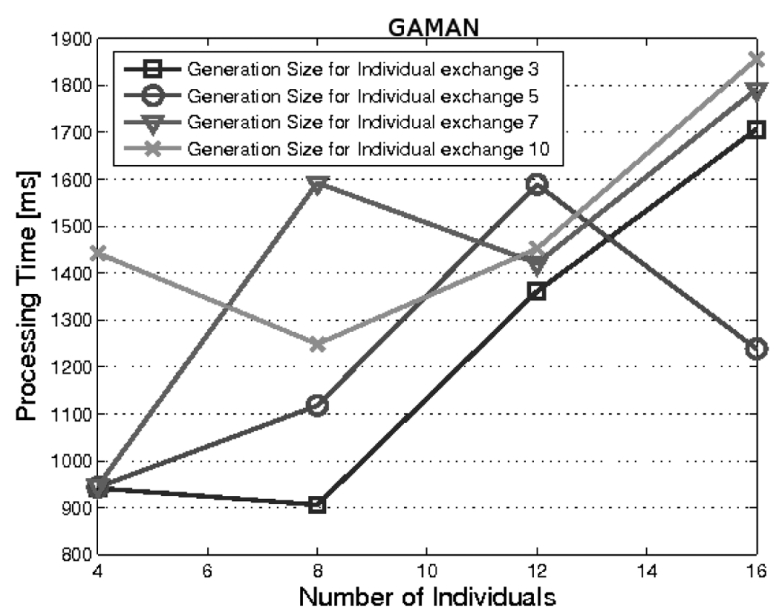

(b) Processing time

Fig. 18. Relation between generation size and number of individuals. (Colours are visible in the online version of the article; http://dx.doi.org/10.3233/MIS-2011-0116)

\section{Conclusions}

In this paper we propose a new method for Ad-hoc networks considering GA and multi-objective optimization. The proposed method uses the multi-division group model for multi-objective optimization.

We evaluated SSRA and GAMAN by computer simulations. From the simulation results, we found the following results.

- The SSRA can reduce the search space of GAMAN, so GAMAN can find faster a new route.

- With increase of mutation rate, the average number of generations and average processing time is decreased. But, with increase of crossover rate, the average number of generations and average processing time is increased. However, when the mutation rate is $8 \%$, the performance of proposed method is better than $10 \%$. Our proposed method has the best performance for crossover rate $70 \%$ and mutation rate $8 \%$.

- If there are few individuals in the population, the GS which shows the number of generations needed to find a solution becomes large.

- GAMAN has better performance than GLBR.

We would like to consider in the future a policy-based routing using FL. We want to put the policies in the Fuzzy Rule Base (FRB) and use this policies for QoS-routing. We also want to investigate an integrated approach of GA-based routing and policy-based routing. We also want to consider the swarm intelligence approach combined with GA for QoS routing in Ad-hoc networks. Population of 
the agents (ants) can be adapted according to the problem size. In swarm intelligence the scalability can be promoted by local and distributed agent interactions. Swarm intelligent processes do not rely on a centralized control mechanism. Therefore the loss of a few agents does not result in catastrophic failure, but rather leads to graceful, scalable degradation. Agents can change, die or reproduce, according to system changes.

\section{References}

[1] C.E. Perkins, Ad-hoc Networking, Addison-Wesley, 2001.

[2] E.M. Royer and C.K. Toh, A Review of Current Routing Protocols for Ad-hoc Mobile Wireless Networks, IEEE Personal Communications (April 1999), 46-55.

[3] S. Chen and K. Nahrstedt, An Overview of Quality of Service Routing for Next-Generation High-Speed Networks: Problems and Solutions, IEEE Network, Special Issue on Transmission and Distribution of Digital Video, Vol. 12, No. 6, pp. 64-79, 1998.

[4] W.C. Lee, M.G. Hluchyj and P.A. Humblet, Routing Subject to Quality of Services Constraints in Integrated Communications Networks, IEEE Network 9(4) (1995), 46-55.

[5] H. Xiao, W.K.G. Seah, A. Lo and K.C. Chua, A Flexible Quality of Service Model for Mobile Ad-hoc Networks, Proc. of IEEE VTC-2000, Tokyo, May 2000.

[6] G.S. Ahn, A.T. Campbell, A. Veres and L.H. Sun, Supporting Service Differentiation for Real-Time and Best-Effort Traffic in Stateless Wireless Ad-hoc Networks (SWAN), IEEE Transactions on Mobile Computing 1(3) (2002), $192-207$.

[7] S.B. Lee, G.S. Ahn, X. Zhang and A.T. Campbell, INSIGIA: An IP-Based Quality of Service Framework for Mobile Ad-hoc Networks, Journal of Parallel and Distributed Computing 60 (2000), 374-406.

[8] S. Chen, S.H. Shah and K. Nahrstedt, Cross-Layer Design for Data Accessibility in Mobile Ad-hoc Networks, Journal of Wireless Personal Communications 21 (2001), Special Issue on Multimedia Network Protocols and Enabling Radio Technologies, Kluwer Academic Publishers, 49-75.

[9] S. Chen and K. Nahrstedt, Distributed Quality-of-Service Routing in Ad-hoc Networks, IEEE Journal of Selected Areas in Communications 17(8) (1999), 1-18.

[10] R. Sivakumar, P. Sinha and V. Bharghavan, CEDAR: a Core-Extraction Distributed Ad-hoc Routing Algorithm, IEEE Journal of Selected Areas in Communications 17(8) (1999), 1454-1465.

[11] K. Wu and J. Harms, QoS Support in Mobile Ad-hoc Networks, Crossing Boundaries - An Interdisciplinary Journal 1(1) (Fall 2001), 92-106.

[12] Special Issue on Computational Intelligence in Telecommunication Networks, Computer Communications 25(16) (2002).

[13] L. Barolli, A Speed-Aware Handover System for Wireless Cellular Networks Based on Fuzzy Logic, Mobile Information Systems (MIS) 4(1) (2008), 1-12.

[14] J. Anno, L. Barolli, A. Durresi, F. Xhafa and A. Koyama, Performance Evaluation of Two-Fuzzy based Cluster Head Selection Systems for Wireless Sensor Networks, Mobile Information Systems (MIS) 4(4) (December 2008), 297-312.

[15] Gj. Mino, L. Barolli, F. Xhafa, A. Durresi and A. Koyama, Implementation and Performance Evaluation of Two Fuzzybased Handover Systems for Wireless Cellular Networks, Mobile Information Systems (MIS) 5(4) (2009), 339-361.

[16] E. Kulla, M. Hiyama, M. Ikeda, L. Barolli, V. Kolici and R. Miho, MANET Performance for Source and Destination Moving Scenarios Considering OLSR and AODV Protocols, Mobile Information Systems (MIS) 6(4) (December 2010), 325-339.

[17] L. Hanzo (II.) and R. Tafazolli, A Survey of QoS Routing Solutions for Mobile Ad-hoc Networks, IEEE Communications Surveys and Tutorials 9(2) (2nd Quarter 2007), 50-70.

[18] R. Gupta, Z. Jia, T. Tung and J. Walrand, Interference-aware QoS Routing (IQRouting) for Ad-hoc Networks, Proc. of Global Telecommunications Conference 5 (November 2005), 2599-2604.

[19] Z. Fan, QoS Routing Using Lower Layer Information in Ad-hoc Networks, Proc. of Personal, Indoor and Mobile Radio Communications Conference, pp. 135-139, September 2004.

[20] D.E. Goldberg, Genetic Algorithms in Search, Optimization, and Machine Learning, Addison-Wesley, 1989.

[21] L. Barolli, A. Koyama, T. Suganuma and N. Shiratori, GAMAN: A GA Based QoS Routing Method for Mobile Ad-hoc Networks, Journal of Interconnection Networks (JOIN) 4(3) (September 2003), 251-270.

[22] M. Munetomo, Y. Takai and Y. Sato, An Adaptive Routing Algorithm with Load Balancing by a Genetic Algorithm, Trans of IPSJ 39(2) (1998), 219-227.

[23] J. Anno, L. Barolli, A. Durresi, F. Xhafa and A. Koyama, Performance Evaluation of Two Fuzzy-based Cluster Head Selection Systems for Wireless Sensor Networks, Mobile Information Systems 4(4) (December 2008), 297-312. 
[24] L. Barolli, M. Ikeda, G. De Marco, A. Durresi, A. Koyama and J. Iwashige, A Search Space Reduction Algorithm for Improving the Performance of a GA-based Routing Method in Ad-hoc Network, International Journal of Distributed Sensor Networks (IJDSN) 3(1) (January 2007), 41-57.

[25] S. Zhao and D. Raychaudhuri, Policy-based Adaptive Routing in Mobile Ad-hoc Wireless Networks, Proc. IEEE Sarnoff-

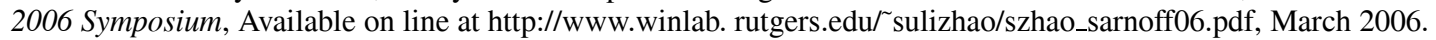

[26] E. Bonabeau, M. Dorigo and G. Theraulaz, Swarm Intelligence: from Natural to Artificial Systems, Oxford University Press, 1999.

[27] M. Dorigo, V. Maniezzo and A. Colorni, The Ant System: Optimization by a Colony of Cooperating Agents, IEEE Transactions on Systems, Man, and Cybernetics, Part B 26(1) (1996), 29-41.

[28] A. Koyama, L. Barolli, G. Capi, B.O. Apduhan, J. Arai and A. Durresi, An Efficient Multi-Purpose Optimization Method for QoS Routing Using Genetic Algorithm, Journal of Interconnection Networks (JOIN) 5(4) (December 2004), 409-428.

[29] Y.C. Hu and D.B. Johnson, Caching Strategies in On-demand Routing Protocols for Wireless Ad-hoc Networks, Proc. of IEEE/ACM MobiCom-2000, 2000.

\begin{abstract}
Admir Barolli was graduated from Agricultural University of Tirana, Albania. He got his Diploma Degree in April 2008. From October 2009 to June 2010, he was a Visiting Researcher at Curtin University of Technology, Australia. Presently, he is a Visiting Researcher at the Department of Computer and Information Science, Seikei University, Japan and working toward his $\mathrm{PhD}$ Degree. He has been working for many years in Agriculture Sectors in Albania. His research interest are in genetics, genetic algorithms, agricultural engineering, intelligent algorithms, climate change, global warming, computer networks and P2P systems.
\end{abstract}

Evjola Spaho received her B.S and M.S degrees at Faculty of Information Technology, Polytechnic University of Tirana (PUT) in 2008 and 2010, respectively. Presently, she is a Ph.D Student at Graduate School of Engineering, Fukuoka Institute of Technology (FIT), Japan. Her research interests include P2P networks, vehicular networks, ad-hoc networks and robot control.

Leonard Barolli is a Professor at the Department of Information and Communication Engineering, Fukuoka Institute of Technology (FIT), Japan. He received BE and PhD Degrees from Tirana University and Yamagata University in 1989 and 1997, respectively. He has published about 300 papers in Journals, Books and International Conference. He has served as a Guest Editor for many Journals. He was PC Chair of IEEE AINA-2004 and ICPADS-2005. He was General Co-Chair of IEEE AINA-2006, AINA-2008 and AINA-2010. He is Steering Committee Chair of CISIS International Conference. His research interests include, P2P, intelligent algorithms, ad-hoc and sensor networks. He is a member of IEEE, IEEE Computer Society, IPSJ and SOFT.

Fatos Xhafa joined the Department of Languages and Informatics Systems of the Technical University of Catalonia as an Assistant Professor in 1996 and is currently an Associate Professor and member of the ALB- COM Research Group of this department. His current research interests include parallel algorithms, combinatorial optimisation, approximation and metaheuristics, distributed programming, grid and P2P computing. He has published in leading international journals and conferences and has served in the organising committees of many conferences and workshops. He is also a member of the editorial board of several international journals.

Makoto Takizawa is a Professor at the Department of Computer and Information Science, Seikei University. He was a Professor and the Dean of the Graduate School of Science and Engineering, Tokyo Denki University. He was a Visiting Professor at GMD-IPSI, Keele University, and Xidian University. He was on the Board of Governors and a Golden Core member of IEEE CS and is a fellow of IPSJ. He received his DE in Computer Science from Tohoku University. He chaired many international conferences like IEEE ICDCS, ICPADS, and DEXA. He founded IEEE AINA. His research interests include distributed systems and computer networks. 

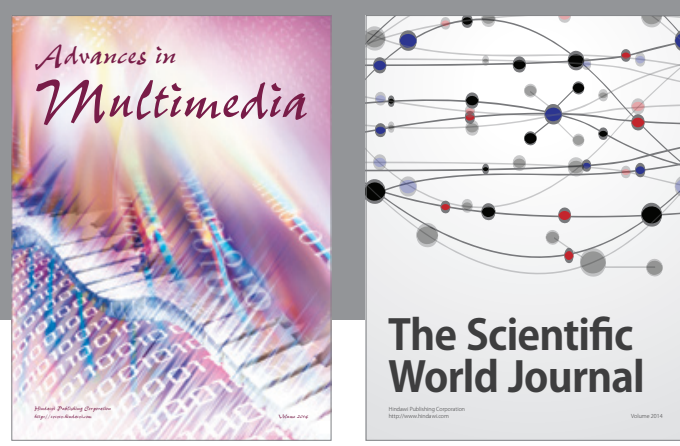

The Scientific World Journal
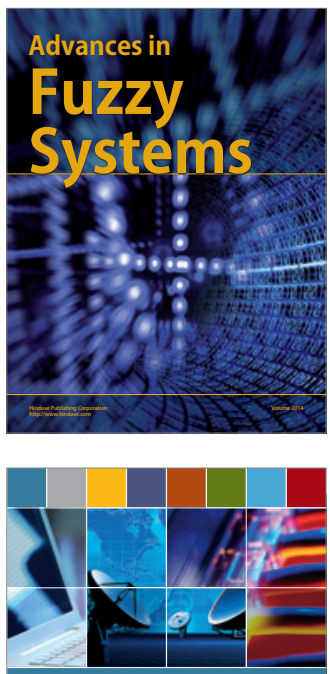

Computer Networks and Communications
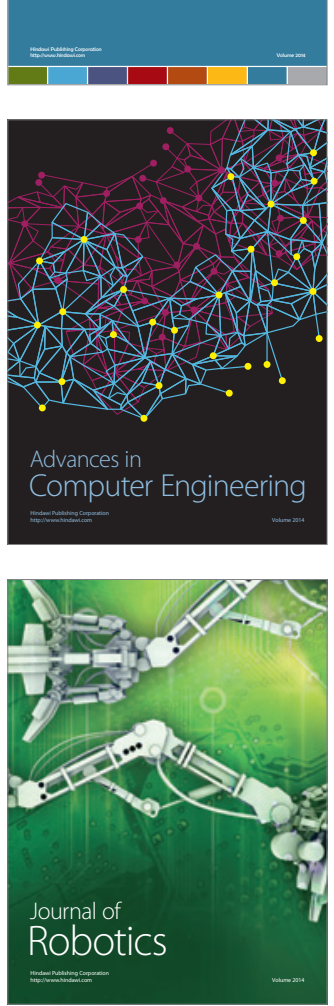
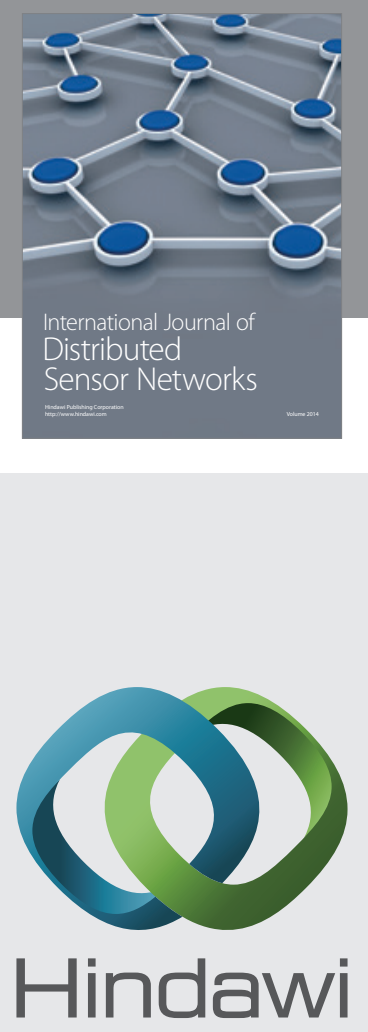

Submit your manuscripts at

http://www.hindawi.com
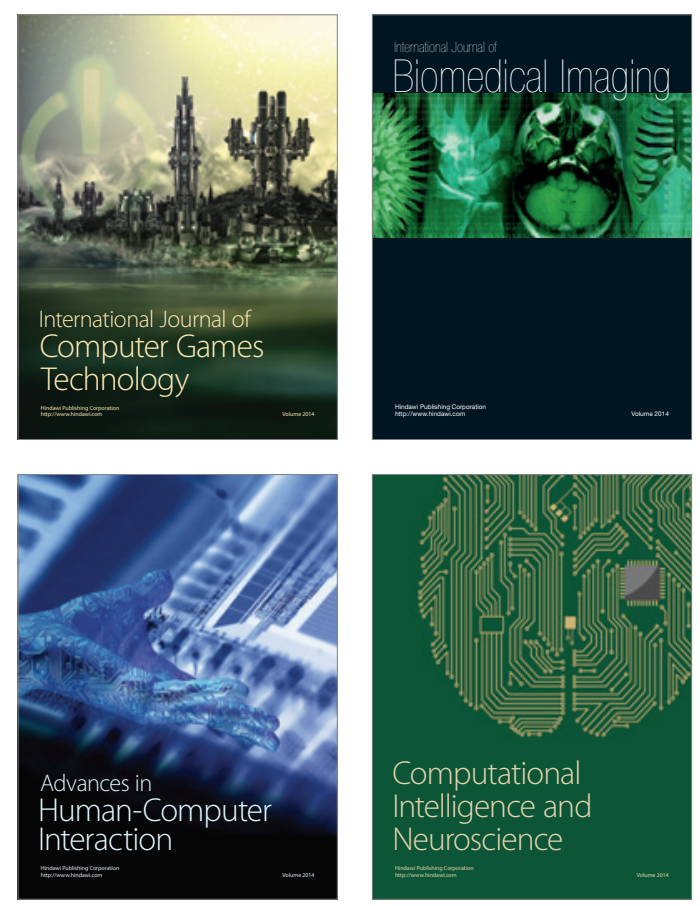
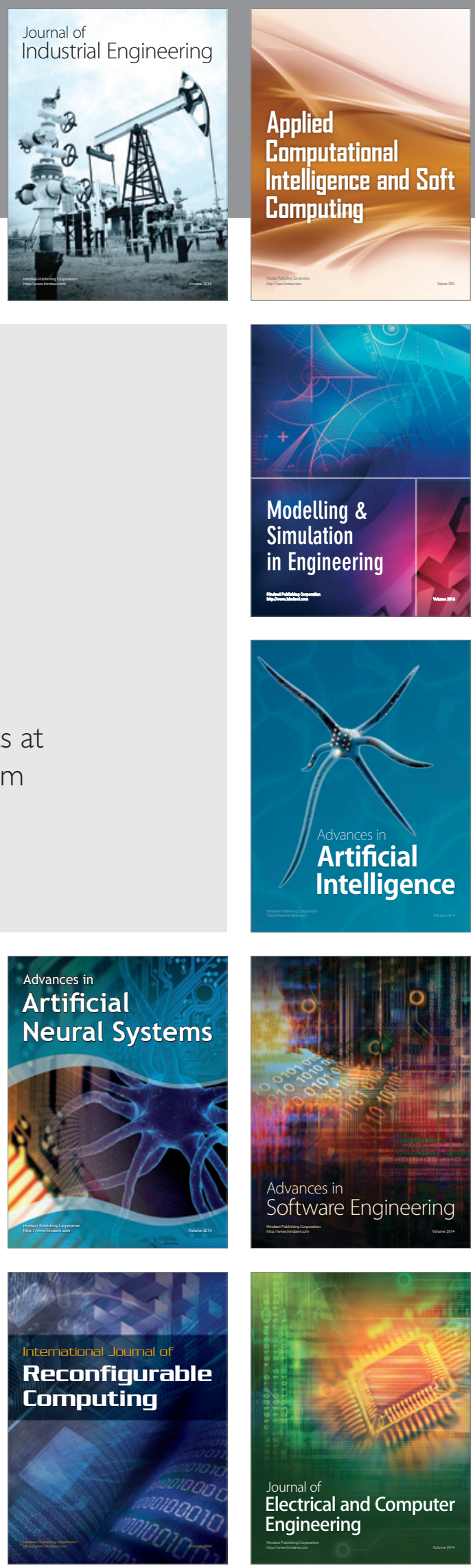